\title{
A Critical Gating Switch at a Modulatory Site in Neuronal Kir3 Channels
}

\author{
Scott K. Adney, Junghoon Ha, Xuan-Yu Meng, Takeharu Kawano, and ๑Diomedes E. Logothetis \\ Department of Physiology and Biophysics, School of Medicine, Virginia Commonwealth University, Richmond, Virginia 23298
}

Inwardly rectifying potassium channels enforce tight control of resting membrane potential in excitable cells. The Kir3.2 channel, a member of the Kir3 subfamily of G-protein-activated potassium channels (GIRKs), plays several roles in the nervous system, including key responsibility in the $\mathrm{GABA}_{\mathrm{B}}$ pathway of inhibition, in pain perception pathways via opioid receptors, and is also involved in alcoholism. PKC phosphorylation acts on the channel to reduce activity, yet the mechanism is incompletely understood. Using the heterologous Xenopus oocyte system combined with molecular dynamics simulations, we show that PKC modulation of channel activity is dependent on Ser-196 in Kir3.2 such that, when this site is phosphorylated, the channel is less sensitive to PKC inhibition. This reduced inhibition is dependent on an interaction between phospho-Ser (SEP)-196 and Arg-201, reducing Arg-201 interaction with the sodium-binding site Asp-228. Neutralization of either SEP-196 or Arg-201 leads to a channel with reduced activity and increased sensitivity to PKC inhibition. This study clarifies the role of Ser-196 as an allosteric modulator of PKC inhibition and suggests that the SEP-196/Arg-201 interaction is critical for maintaining maximal channel activity.

Key words: GIRK; Kir3; PIP ${ }_{2}$; PKC

\section{Significance Statement}

The inwardly rectifying potassium 3.2 (Kir3.2) channel is found principally in neurons that regulate diverse brain functions, including pain perception, alcoholism, and substance addiction. Activation or inhibition of this channel leads to changes in neuronal firing and chemical message transmission. The Kir3.2 channel is subject to regulation by intracellular signals including sodium, G-proteins, ethanol, the phospholipid phosphatidylinositol bis-phosphate, and phosphorylation by protein kinases. Here, we take advantage of the recently published structure of Kir3.2 to provide an in-depth molecular view of how phosphorylation of a specific residue previously thought to be the target of $\mathrm{PKC}$ promotes channel gating and acts as an allosteric modulator of PKC-mediated inhibition.

\section{Introduction}

Inwardly rectifying potassium (Kir) channels are critically important for regulating resting membrane potential in excitable cells, a role underscored by the severe pathophysiology associated

Received April 12, 2015; revised Aug. 20, 2015; accepted Sept. 18, 2015.

Author contributions:S.K.A., X.-Y.M., and D.E.L. designed research;S.K.A., J.H., and T.K. performed research; T.K. contributed unpublished reagents/analytic tools; S.K.A. and X.-Y.M. analyzed data; S.K.A. and D.E.L. wrote the paper.

This work was supported by the National Institutes of Health (Grants R01HL59949 and R01HL090882 to D.E.L.). We thank Heikki Vaananen for Xenopus oocyte isolation and members of the Logothetis laboratory for insightful discussions and comments throughout this project.

The authors declare no competing financial interests.

Correspondence should be addressed to Diomedes E. Logothetis, Department of Physiology and Biophysics, Virginia Commonwealth University School of Medicine, 1101 E Marshall St, P.0. Box: 980551, Richmond, VA 232980551.E-mail: delogothetis@vcu.edu.

X.-Y. Meng's present address: School for Radiological and Interdisciplinary Sciences (RAD-X), Collaborative Innovation Center of Radiation Medicine of Jiangsu Higher Education Institutions, and Jiangsu Provincial Key Laboratory of Radiation Medicine and Protection, Soochow University, Suzhou 215123, China.

DOI:10.1523/JNEUROSCI.1415-15.2015

Copyright $\odot 2015$ the authors $\quad 0270-6474 / 15 / 3514397-09 \$ 15.00 / 0$ with Kir channel dysfunction (Pattnaik et al., 2012). Although all Kir channels require the activating lipid phosphatidylinositol bisphosphate $\left(\mathrm{PIP}_{2}\right)$ for proper function, many of these channels have diverse modulatory factors that couple to $\mathrm{PIP}_{2}$-dependent gating. Channels in the Kir3 subfamily of G-protein-activated potassium channels (GIRKs) in particular have several coactivating intracellular factors, including the $\beta \gamma$ subunits of G-proteins, ethanol, and sodium (Hibino et al., 2010). Moreover, phosphorylation by PKA and PKC are thought to modulate channel activity, providing additional ways of fine-tuning channel gating.

A particular subtype of the Kir3 family, Kir3.2, is primarily expressed in neurons, where it is thought to be responsible for $\mathrm{GABA}_{\mathrm{B}}$-mediated decreases in cellular excitability. Kir3.2 knockout animals have a reduction in opioid-induced hyperpolarization in locus coeruleus neurons, suggesting a role in pain perception (Torrecilla et al., 2002). Homotetramers of Kir3.2 may exist in the substantia nigra pars compacta, where there does not appear to be expression of its usual heteromeric partner, Kir3.1 (Inanobe et al., 1999). Kir3.2 is readily activated by G $\beta \gamma$ 
via Gi-coupled GPCRs (Logothetis et al., 1987; Hibino et al., 2010), whereas alternate pathways of control, such as channel inhibition via Gq-coupled GPCRs, are less well understood (Kobrinsky et al., 2000; Leaney et al., 2001; Brown et al., 2005; Hibino et al., 2010).

The activation of Gq-coupled receptors triggers PIP $_{2}$ hydrolysis and generation of $\mathrm{IP}_{3} / \mathrm{Ca}^{2+}$, as well as DAG signals, both of which synergistically activate multiple PKC isoforms. The net downstream effect of PKC activation on Kir3 channels is current inhibition, allowing for increased cellular excitability (Stevens et al., 1999; Mao et al., 2004; Keselman et al., 2007). Gq-coupled receptor activation also simultaneously depletes the local concentration of $\mathrm{PIP}_{2}$ by hydrolysis to $\mathrm{IP}_{3}$, which decreases Kir3 channel activity because $\mathrm{PIP}_{2}$ is a required component for activation of Kir3 channels (Huang et al., 1998; Zhang et al., 1999). The combination of PKC activation and $\mathrm{PIP}_{2}$ depletion acts in concert to further inhibit Kir3 channel activity. Accumulating evidence suggests that PKC-mediated phosphorylation inhibits channel activity by reducing its apparent affinity for $\mathrm{PIP}_{2}$, in effect changing the level of $\mathrm{PIP}_{2}$ available for the channel to sense (Zhang et al., 2004; Keselman et al., 2007; Sohn et al., 2007a).

A presumed phosphorylation site, identified by functional site-directed mutagenesis on the Kir3.1 and Kir3.4 isoforms, localizes on the Kir3.2 channel to Ser-196 (Mao et al., 2004). This residue is located near the bottom of the helix-bundle-crossing

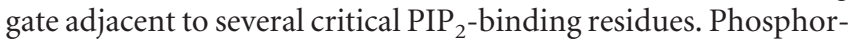
ylation of this residue would likely affect channel-gating dynamics by perturbing local electrostatic interactions involved in channel-PIP ${ }_{2}$ interactions. Ser-196 also lies in close proximity to the KKR motif, a highly conserved region in Kir channels contributing directly to $\mathrm{PIP}_{2}$ binding. This was previously suggested by neutralization mutagenesis techniques (Lopes et al., 2002) and has recently been confirmed by $\mathrm{x}$-ray crystallography (Hansen et al., 2011; Whorton and MacKinnon, 2011). The Arg residue at the distal end of the KKR motif has recently been assigned a critical gating role in the Kir3.2 channel, whereby the neutralization mutant R201A was suggested to create a constitutively active channel, mimicking $\mathrm{G} \beta \gamma$ subunit binding (Whorton and MacKinnon, 2011; Whorton and MacKinnon, 2013).

Given the juxtaposition of Ser-196 to several critical gating elements of the Kir3.2 channel, together with the information afforded by the recently available crystal structure of Kir3.2 bound to $\mathrm{PIP}_{2}$, we sought to examine in greater depth how phosphorylation at Ser-196 could lead to changes in PIP $_{2}$-mediated gating. To accomplish this, we combined computational molecular dynamics simulations with mutagenesis techniques using results from the simulations to guide our experiments. We found that neutralization of Ser-196 allosterically increased PKC inhibition, but in a $\mathrm{PIP}_{2}$-independent manner. When phosphorylated, this serine residue forms a critical interaction with Arg-201, disrupting an Arg-201/Asp-228 salt bridge.

\section{Materials and Methods}

Molecular biology. For expression in Xenopus oocytes, the following vectors were used: pGEMHE (for Kir3.4), pXoom (for Kir3.2), and pSD64TF (for Ci-VSP). Point mutations were introduced using standard $P f u$-based mutagenesis technique according to the QuikChange protocol (Stratagene) and mutations were verified by sequencing (Genewiz). Plasmids were linearized before in vitro transcription using a commercially available mRNA transcription kit (Ambion).

Oocyte preparation and injection. Oocytes from female X. laevis were surgically removed and subjected to collagenase treatment according to standard protocols. Once defolliculated, oocytes were transferred to OR2 containing the following (in $\mathrm{mM}$ ): $2 \mathrm{KCl}, 82.5 \mathrm{NaCl}, 2 \mathrm{MgCl}_{2}$, and 10
HEPES-Na, $\mathrm{pH} 7.5$, supplemented with $\mathrm{Ca}^{2+}$ and penicillin/streptomycin antibiotics. Stage V or VI oocytes were injected with $50 \mathrm{nl}$ of cRNA resuspended in DEPC water. For wortmannin treatments, oocytes were incubated in wortmannin-containing OR2 (without antibiotics) at a $50 \mu \mathrm{M}$ final concentration for $2 \mathrm{~h}$ before experiments. For Bis treatment, $30 \mathrm{nl}$ of $150 \mu \mathrm{M}$ Bis (diluted in ND96K) was injected into oocytes $2 \mathrm{~h}$ before experiments. Oocytes were also incubated in $5 \mu \mathrm{M}$ Bis (in OR2 media).

Two-electrode voltage clamp. Borosilicate glass electrodes were pulled using a Flaming-Brown micropipette puller (Sutter Instruments) and filled with a $3 \mathrm{M} \mathrm{KCl}$ solution containing 1.5\% agarose. Resistances were kept between 0.3 and $1.0 \mathrm{M} \Omega$. Currents were recorded $1-3 \mathrm{~d}$ after injection using a GeneClamp 500 amplifier (Axon). A voltage-ramp protocol was used to monitor inward current, from $-80 \mathrm{mV}$ to $+80 \mathrm{mV}$ at a holding of $0 \mathrm{mV}$. Specialized Ci-VSP protocols were used to monitor the impact of $\mathrm{PIP}_{2}$ depletion on channel current. To obtain the percentage of PMA inhibition, the PMA-inhibited current at $-80 \mathrm{mV}\left(I_{\max }-\right.$ $\left.I_{\text {post-PMA }}\right)$ was divided by the maximal current at $-80 \mathrm{mV}\left(I_{\max }\right)$ before PMA application. In channels with very small currents $(<1 \mu \mathrm{A}$, as in Kir3.2WT or Kir3.2_I234L_196Q), the raw current was first barium subtracted to exclude endogenous inward currents from the analysis, which were typically $200-300 \mathrm{nA}$ at $-80 \mathrm{mV}$. Barium subtraction was then applied to all members of the test group. The ND96K (HK) solution used to monitor inward current contained the following (in $\mathrm{mM}$ ): $96 \mathrm{KCl}, 10$ HEPES-K, $1 \mathrm{MgCl}_{2}$, and $1.8 \mathrm{CaCl}_{2}$, pH 7.4. ND96 (LK) contained the following (in mM): $96 \mathrm{NaCl}, 2 \mathrm{KCl}, 5 \mathrm{HEPES}-\mathrm{Na}, 1 \mathrm{MgCl}_{2}$, and $1.8 \mathrm{CaCl}_{2}$, $\mathrm{pH}$ 7.4. PMA was dissolved in DMSO to make a $10 \mathrm{~mm}$ stock and diluted into ND96K for a $300 \mathrm{~nm}$ final concentration. For barium block, a solution of $5 \mathrm{~mm} \mathrm{BaCl}_{2}$ in ND96K was perfused.

Protein purification. Mouse Kir3.2C(1-425aa)-GFP-His10 cDNA was constructed in the Pichia expression vector pPICz (Invitrogen). The X-33 strain was transformed with the PmeI-linearized plasmid by electroporation. Protein expression was induced with $0.5 \%$ methanol-containing BMMY medium at $25^{\circ} \mathrm{C}$ for $24 \mathrm{~h}$. Kir3.2C protein was purified according to Whorton and MacKinnon (2011). Briefly, membrane proteins were prepared by ultracentrifugation (for $30 \mathrm{~min}$ at $100,000 \times \mathrm{g}$ ) after cell disruption using EmulsiFlex-C3 homogenizer (Avestin). Kir3.2C-GFPHis 10 proteins were solubilized with $4 \%$ DDM (Anatrace). Supernatant of centrifugation (for $20 \mathrm{~min}$ at $40,000 \times g$ ) was subjected to Ni-NTA chromatography (Qiagen). After TEV-cleavage of C terminus GFPHis10 tag, Kir3.2C homotetramer was purified using Superdex-200 column (GE Healthcare) by size-exclusion chromatography.

Alkaline phosphatase digestion. $1 \mu \mathrm{g}$ of purified Kir3.2 protein or $1 \mu \mathrm{g}$ of $\beta$-casein (Sigma-Aldrich) was combined with 10 units of calf intestinal phosphatase (New England Biolabs) in $1 \times$ NEB Buffer 3 and incubated $1 \mathrm{~h}$ at $37^{\circ} \mathrm{C}$. The reaction was terminated with $4 \times$ SDS sample buffer and samples were boiled and loaded onto an SDS-PAGE gel. The gel was stained with Pro-Q Diamond Phosphoprotein Gel Stain (Invitrogen) according to the manufacturer's instructions. Briefly, the gel was fixed overnight with $50 \%$ methanol, $10 \%$ acetic acid solution, washed $4 \times 15$ min with MilliQ water, and stained for $2 \mathrm{~h}$ with ProQ PhosphoStain. The staining step and all steps thereafter were performed in the dark. The gel was destained $4 \times 30 \mathrm{~min}$ with a solution containing $50 \mathrm{~mm} \mathrm{Na}$-acetate, pH 4.0, and $20 \%$ acetonitrile. The gel was washed $2 \times 10$ min with MilliQ water before visualization on a Typhoon fluorescent image scanner set to excitation at $532 \mathrm{~nm}$ and emission at $560 \mathrm{~nm}$.

Molecular dynamics. For simulations, systems were set up similar to a previously described method (Meng et al., 2012). Briefly, a channel tetramer was generated from the crystal coordinates of PDB code 3SYA, corresponding to Kir3.2 with DiC8-PIP ${ }_{2}$ and sodium. The acyl chains of native $\mathrm{PIP}_{2}$ (20:4, arachidonyl-stearyl) were manually built with Discovery Studio onto the crystal $\mathrm{PIP}_{2}$ head group. The tetramer was inserted into a POPC membrane via the INFLATE script. The system was solvated (SPC model) and ionized $(0.15 \mathrm{KCl})$ in GROMACS and then sodium ions and potassium ions were manually added to the sodium site and the selectivity filter, respectively, by superimposition using the 3SYA structure. The 53a6 GROMOS force-field was altered to include parameters for $\mathrm{PIP}_{2}$ and phosphoserine (SEP). After minimization using a steepest descent algorithm, two equilibration runs were executed with progres- 
A
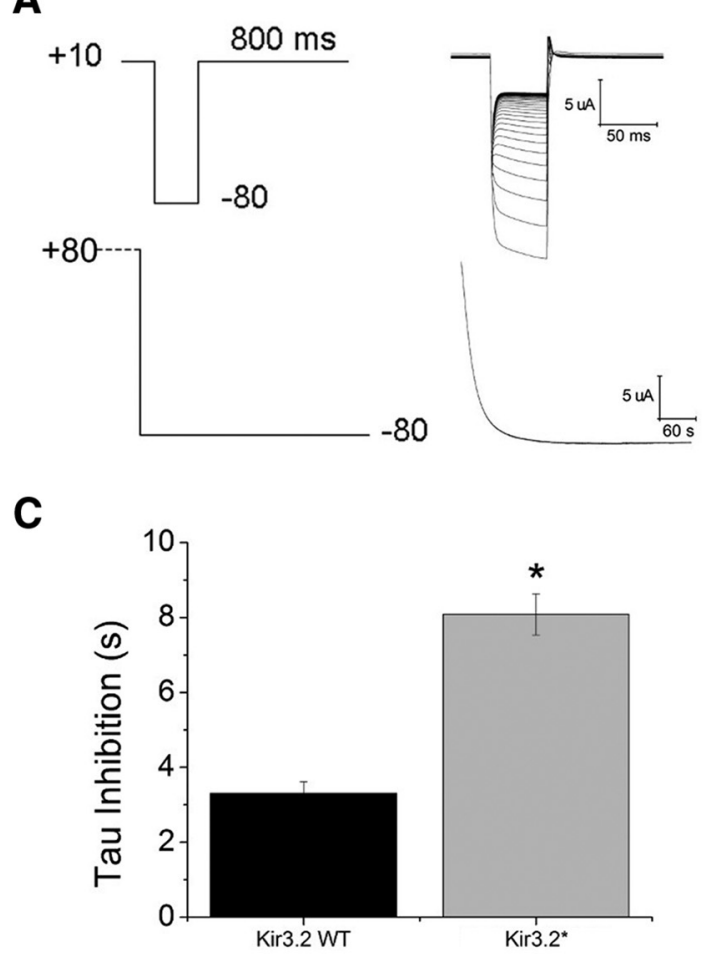

B

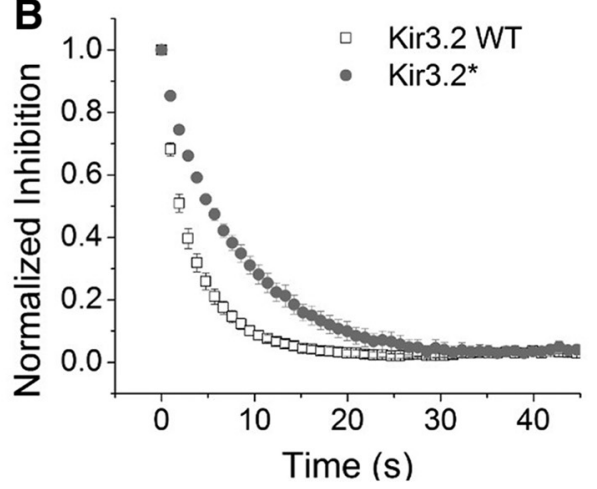

D

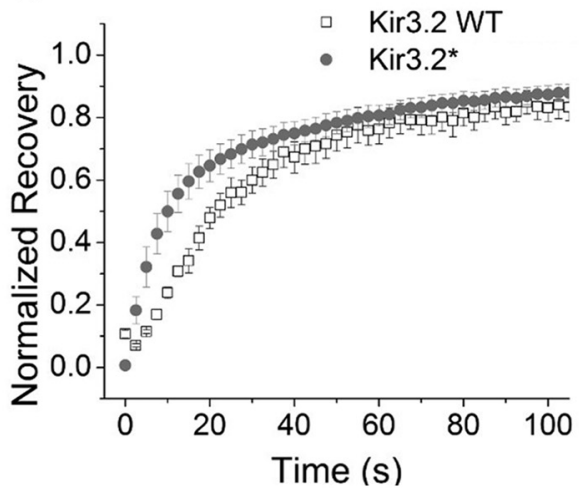

Figure 1. Kir3.2* (Kir3.2_E152D) has slower Ci-VSP-mediated inhibition and faster recovery versus wild-type Kir3.2. A, Left, Voltage protocols (in mV) used for inhibition (top) and recovery (bottom). Right, Sample inhibition traces for Kir3.2* (top) and recovery (bottom). B, Kir3.2* displays slower current inhibition compared with wild-type Kir3.2. C, Tau of inhibition for Kir3.2* is significantly slower than wild-type. ${ }^{*} p<0.05$ compared with control. $\boldsymbol{D}$, Kir3.2* has faster recovery than wild-type Kir3.2 at $-80 \mathrm{mV}$.

sively weaker position restraint (1000 then 10$)$ on heavy atoms, followed by a 100 ns production run with no restraints. Simulation analysis was run using the GROMACS, Simulaid, and VMD programs.

Statistics. All error bars represent the SEM. Statistical significance was assessed using Student's $t$ test assuming unequal variance between samples in Origin 8.5 (OriginLab). Statistical significance was set at $p<0.05$, which is denoted by an asterisk in figures. For Ci-VSP-mediated current inhibition, a single-component exponential function was fit to the descending phase of the data and the tau was extracted. For normalized current data, barium-subtracted currents for each group were divided by the mean of the control group. All experiments were repeated in at least two separate batches of oocytes.

\section{Results}

Homomeric Kir channel expression often leads to low currents when expressed in heterologous systems. To overcome this, pore mutants that increase channel activity are usually used (Vivaudou et al., 1997). In the case of Kir3.2, the E152D mutant was identified in a yeast expression assay, boosting current levels by increasing channel activity (NPo) (Yi et al., 2001). We hypothesized that the increase in NPo may be due to an allosteric enhancement in channel-PIP 2 interactions and used the voltage-gated lipid phosphatase Ci-VSP to test this hypothesis. As shown in Figure 1, the Kir3.2_E152D (hereafter referred to as Kir3.2*) mutant had significantly slower inhibition $\left(8.1 \mathrm{~s} \tau_{\text {Inhibition }}\right.$ for Kir3.2* $[n=6]$ vs 3.3 s for Kir3.2 $[n=$ $5], p=0.00005)$ and faster recovery than the wild-type Kir3.2 channel. These results are consistent with Kir3.2* having increased channel-PIP 2 interactions (slower inhibition and faster recovery kinetics) compared with the wild-type Kir3.2.

We next compared the extent of PMA inhibition between two different homomeric pore-mutant channels, Kir3.4* (S143T)
(Vivaudou et al., 1997) and Kir3.2*. We perfused 300 nм PMA, a membrane-permeable PKC activator, and quantified the extent of inhibition for Kir3 homomeric channels. To determine the extent of inhibition, the PMA-sensitive current $\left(I_{\max }-I_{\text {post-PMA; }}\right.$ double arrows in Fig. 2) was divided by the current before PMA application ( $I_{\max }$; single arrow in Fig. 2 ) and expressed as a percentage. Interestingly, Kir3.2* showed significantly less PMA inhibition when compared with Kir3.4* (Fig. 2A,C,E; Kir3.2* $23.6 \pm 2.3 \%[n=4]$ vs Kir3.4* $72.4 \pm 3.5 \%[n=6], p<0.001)$. Bisindolylmaleimide (Bis), an inhibitor of PKC, abrogated the PMA inhibitory effects on both Kir3 channels (Fig. $2 B, D, E$ ).

To determine whether PMA inhibition was influenced by membrane $\mathrm{PIP}_{2}$ content, we used the drug wortmannin, which can inhibit PI4K at micromolar concentrations (Zhang et al., 2003). When oocytes expressing Kir3.2* were preincubated with $50 \mu \mathrm{M}$ wortmannin for $2 \mathrm{~h}$ to reduce membrane $\mathrm{PIP}_{2}$ content, the extent of PMA inhibition was significantly increased (Kir3.2* control $23.6 \pm 2.3 \%[n=4]$ vs wortmannin-treated $66.1 \pm 1.4 \%$ $[n=5], p=0.000006$ ) (also discussed below in Fig. 4C). These results suggest that the inhibition of Kir3.2* by PMA is influenced by membrane $\mathrm{PIP}_{2}$ content.

Having found that Kir $3.4^{*}$ and Kir3.2* homomeric channels are subject to differing levels of PMA inhibition, we sought to determine whether a previously identified PKC phosphorylation site may be involved in this behavior. We first used Kir3.4* homomeric channels mutated at Ser-191 using mutations to either abrogate (alanine) or to mimic (aspartate) phosphorylation, respectively. Consistent with previously published results in the Kir3.4 channel (Mao et al., 2004), Kir3.4*_S191A had significantly reduced PMA inhibition compared with Kir3.4* 


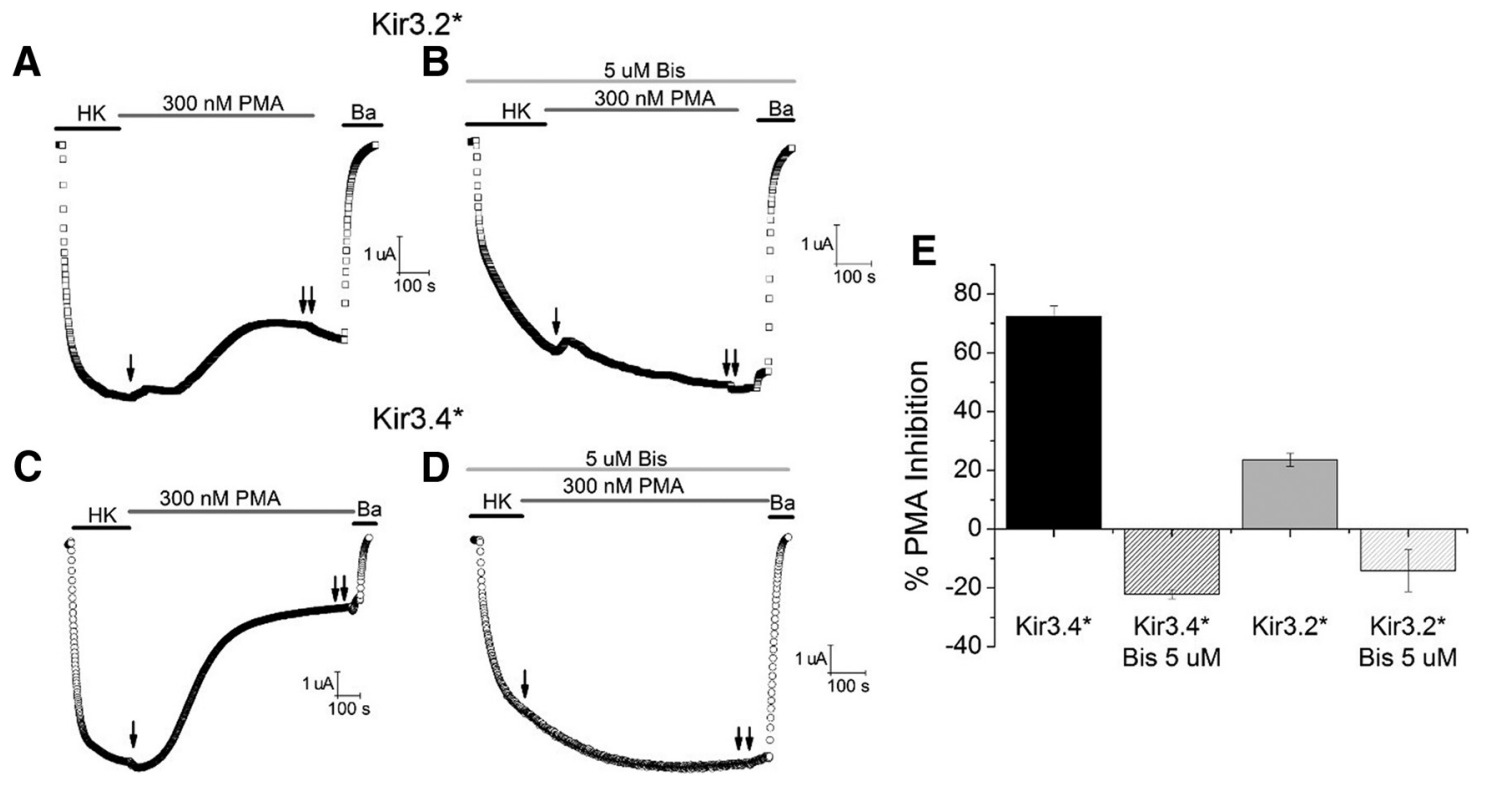

Figure 2. Kir3.2* has lower PMA inhibition relative to Kir3.4* and both PMA effects are blocked by PKC inhibition. A, Time course of PMA inhibition in control Kir3.2_E152D (Kir3.2*).Single arrows indicate $I_{\text {max }}$ and double arrows indicate $I_{\text {post-PMA. }}$ B, Time course of PMA inhibition in Kir3.2* after Bis pretreatment. C, Time course of PMA inhibition in control Kir3.4_S143T (Kir3.4*). D, Time course of PMA inhibition in Kir3.4* after Bis pretreatment. $E$, Summary of PMA inhibition with and without Bis pretreatment.
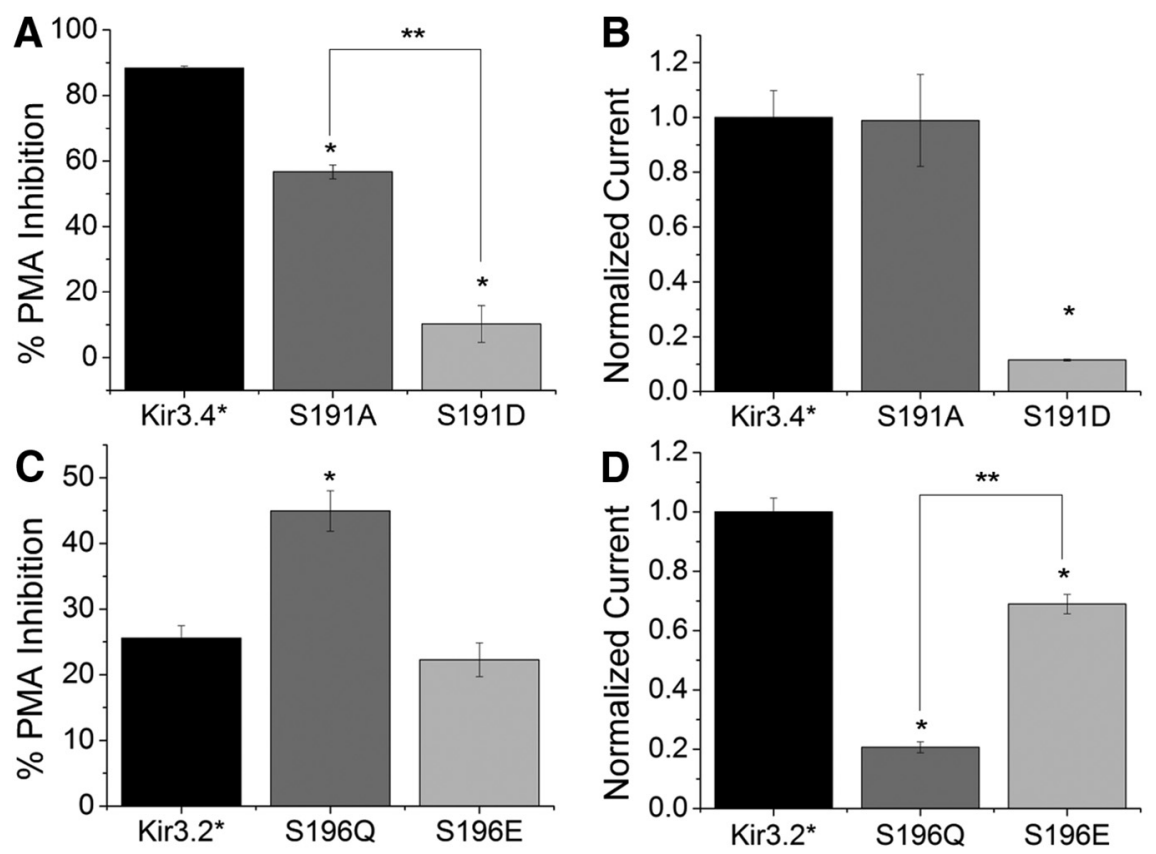

E

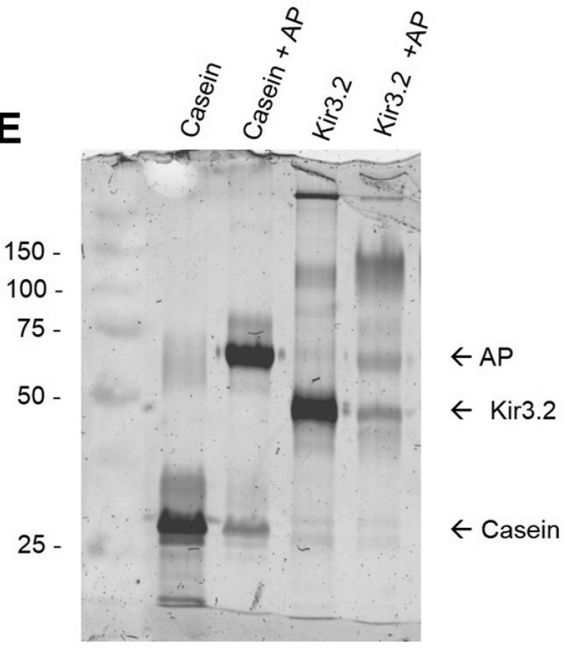

Figure 3. PMA inhibition and normalized currents for Kir3.2* and Kir3.4* phosphorylation site mutants. A, PMA inhibition for Kir3.4* Ser-191 mutants. ${ }^{* *} p<0.05$ between indicated groups; ${ }^{*} p<0.05$ compared with control. B, Normalized currents of Kir3.4* Ser-191 mutants. C, PMA inhibition for Kir3.2* Ser-196 mutants. D, Normalized currents of Kir3.2* Ser-196 mutants. E, Purified Kir3.2 from $P$. pastoris treated with alkaline phosphatase reveals constitutive phosphorylation of the channel protein.

(Kir3.4*_S191A $56.7 \pm 2.1 \%[n=5]$ vs Kir3.4* control $88.3 \pm$ $0.7 \%[n=3],{ }^{*} p<0.05$; Fig. $3 A$ ). The phosphomimetic Kir3.4*_S191D exhibited $10.3 \pm 5.6 \%[n=3]$ inhibition, resulting in a significant reduction compared with Kir3.4*_S191A, as previously reported by Mao et al. (2004) (Fig. 3A).

Interestingly, the pattern of PMA inhibition was reversed in Kir3.2* homomeric channels mutated at the corresponding site, Ser-196. In the Kir3.2* channel, PMA inhibition of the phosphomimetic S196E mutant was not significantly different from Kir3.2* control (Kir3.2* $25.6 \pm 1.9 \%[n=12]$ vs Kir3.2*_S196E $22.3 \pm 2.6 \%[n=11]$; Fig. $3 C)$. Surprisingly, the Kir3.2*_S196Q neutralization mutant had significantly higher PMA inhibition $(44.9 \pm 3.1 \%[n=12], p<0.05)$ than either the Kir3.2* control or S196E. These results indicate that Kir3.2* has increased PMA inhibition after neutralization of Ser-196, compared with Kir3.4*, which has the opposite effect, reduced PMA inhibition.

We then compared current levels of the phosphorylation site mutants, hypothesizing that the total current reflects the degree of channel activity imparted by the mutant. We normalized each mutant to its control channel and recorded the peak current in ND96K solution. Using this method, Kir3.4*_S191A (98 土 $16.7 \%[n=5])$ had similar current levels to Kir3.4* control 

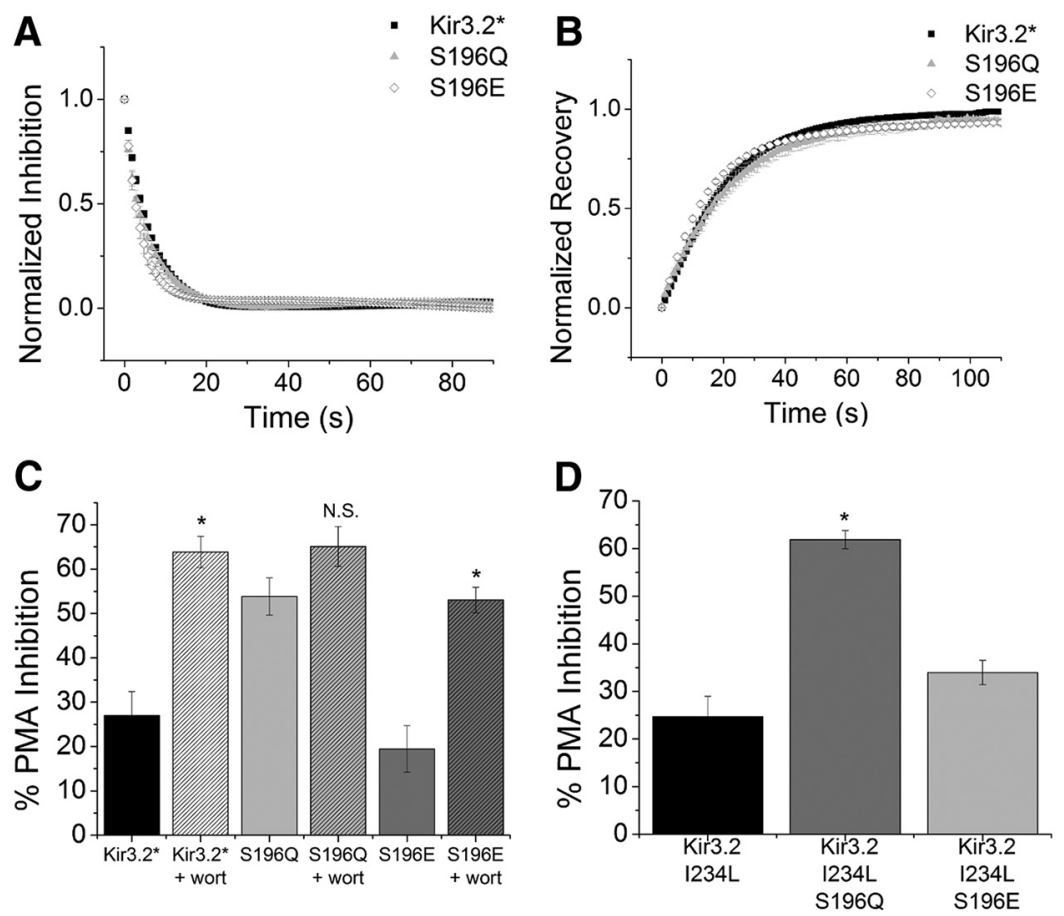

Figure 4. Kir3.2* mutants show similar sensitivity to $\mathrm{PIP}_{2}$ as the Kir3.2* control and PMA sensitivity of $\mathrm{S} 196 \mathrm{Q}$ is independent of $\mathrm{PIP}_{2}$ manipulation. $\boldsymbol{A}$, Normalized Ci-VSP inhibition of Kir3.2 ${ }^{*}$ and Ser-196 mutants. $\boldsymbol{B}$, Normalized recovery of Kir3.2 ${ }^{*}$ and Ser-196 mutants. Protocols for inhibition and recovery were identical to experiments shown in Figure 1. C, PMA inhibition for Kir3.2* Ser-196 mutants in control and with wortmannin pretreatment. ${ }^{*} p<0.05$ compared with control condition. $\boldsymbol{D}$, PMA inhibition of Kir3.2_1234L Ser-196 mutants. ${ }^{*} p<0.05$ compared with Kir3.2_I234L control. N.S., Not significant.

$[n=5]$, whereas Kir3.4*_S191D had significantly diminished currents $(11.5 \pm 0.3 \%[n=5])$ (Fig. $3 B)$. In the Kir3.2* channel, the S196Q mutant was $20 \pm 1.8 \%[n=11]$ of control $[n=9]$, whereas the Kir3.2*_S196E mutant was closer to Kir3.2*, at $69.0 \pm 3 \%[n=13]$ (Fig. $3 D$ ). These results indicate that the effect of residue substitution at this position in different Kir3 channels is heterogeneous. Kir $3.4^{*}$ follows a pattern consistent with the phosphomimetic residue playing a role in PMA inhibition, namely that the S191D mutant has significantly reduced currents, whereas S191A does not change current level. For Kir3.2*, the neutralization mutant S196Q significantly reduces currents, whereas the phosphomimetic S196E has currents that are closer to control, suggesting that the Kir3.2 channel is constitutively phosphorylated at Ser-196.

We proceeded to purify the full-length Kir3.2 protein from Pichia pastoris and test whether it was constitutively phosphorylated by treating with alkaline phosphatase (AP). Using ProQ Diamond Phosphostain, the Kir3.2 phosphoprotein staining was significantly reduced following AP treatment compared with the control conditions, suggesting that purified Kir3.2 is indeed subject to constitutive phosphorylation. Casein, a constitutively phosphorylated protein, was used as a control in this assay (Fig. 3E).

The effects seen with Kir3.2*_S196 mutants were not due to potential changes that these mutations could have had to channel-PIP 2 interactions because the Ci-VSP-induced current inhibition and recovery were identical for Kir3.2* and its S196 mutants (Fig. $4 A, B$ ). In addition, wortmannin pretreatment of Kir3.2*_S196E increased the PMA sensitivity from $19.4 \pm 5.2 \%$ $[n=3$, control $]$ to $53 \pm 2.9 \%[n=4$, wortmannin-treated; $p=$ $0.0018]$, similar to the wortmannin effect on Kir3.2* alone. The S196Q mutant did not follow this pattern because wortmannin pretreatment did not significantly increase PMA inhibition
$(53.8 \pm 4.1 \%[n=4$, control $]$ vs $65.1 \pm$ $4.5 \%[n=4$, wortmannin-treated $] ; p=$ 0.12; Fig. 4C). We also tested the Ser-196 mutations in the background of the $\mathrm{I} 234 \mathrm{~L}$ mutant, which lead to increased channel currents presumably due to increased channel-PIP 2 interactions. These currents were sufficiently large enough to allow analysis in the wild-type Kir3.2 background. As shown in Figure $4 D$, the mutants displayed the same pattern of inhibition in the I234L background, where S196Q had increased inhibition (61.9 \pm $3.8 \%$, Kir3.2_I234L_S196Q $[n=4]$ vs $24.7 \pm 7.4 \%$, Kir3.2_I234L $[n=3] ; p=$ 0.0031) compared with control, whereas S196E did not differ from the control $(33.9 \pm 5.7 \%$, Kir3.2_I234L_S196E $[n=$ $5] ; p=0.09)$. Together, these results demonstrate that Ser-196 mutations influence PKC inhibition in a $\mathrm{PIP}_{2}$-independent manner.

We hypothesized that substitution of the Ser-196 residue may lead to differential effects on gating by perturbing local electrostatic interactions in the channel itself or with the gating molecule $\mathrm{PIP}_{2}$. To test this hypothesis, we began by using molecular dynamics simulations with the recently solved crystal structure of Kir3.2 in complex with $\mathrm{PIP}_{2}$ (PDB code 3SYA) and performed mutations at Ser-196 in silico in the background of the E152D mutant (Kir3.2*), similar to our experimental approach. The Ser-196 residue is located approximately one helical turn from the key gating residue Phe-192, which forms the helix bundle crossing gate. We examined the intersubunit distance of this gate by calculating the minimal distance of residue-residue interactions at Phe-192. Interestingly, the S196E mutant adopts a semi-open conformation at the helix bundle crossing whereby the intersubunit $\mathrm{A}-\mathrm{B}$ distance increases, but the intersubunit C-D distance does not (Fig. 5B). In the S196Q mutant, both F192 distances remain stable and the gates maintain a closed conformation (Fig. 5A).

To better understand the molecular interactions that facilitate the channel adopting this semi-open conformation, we analyzed protein salt-bridge interactions of the S196Q and S196E mutants throughout their respective simulations. In the S196Q mutant, a strong salt-bridge interaction persists between Asp-228 and Arg201 of each subunit. Conversely, in the S196E mutant, the negatively charged Glu-196 recruits Arg-201 away from Asp-228. The carbon-to-carbon distance averaged over the four channel subunits is depicted in Figure 6. Consistent with the salt-bridge results for S196E, the average distance for Arg-201/Asp-228 increases (from $\sim 4 \AA$ to $\sim 8.1 \AA$ ), whereas simultaneously the distance for Arg-201/Glu-196 decreases (from $\sim 10 \AA$ to $\sim 6.5 \AA$ ), allowing interaction (Fig. $6 B$ ). In the S196Q mutant, the average distances for Arg-201/Asp-228 and Arg-201/Gln-196 did not change appreciably over the 100 ns simulation (Fig. 6A).

Inspection of the trajectories and an 80 ns snapshot of the two simulations (Fig. 6C,D) indicates that, in the S196E mutant, Arg-201 moves away from Asp-228 and swings upward to form interactions with Glu-196. In the S196Q mutant, Arg-201 firmly interacts with Asp-228 for the duration of the simulation. The phosphorylated 
channel (Fig. $7 B$ ) and the nonphosphorylated control (Fig. 7A) likewise behaved similarly in the simulation to their mutant counterparts, S196E and S196Q, respectively. Only the phosphorylated serine (SEP196) formed salt-bridge interactions with Arg-201, recruiting the critical residue away from Asp-228, much like the pattern seen with the S196E mutant.

We proceeded to test the outcome of these simulations that suggested a switch mechanism whereby phosphorylation at Ser-196 could recruit Arg-201 away from Asp-228. The mutant R201A that was used as a constitutively active channel was shown to adopt a semiopen state when cocrystallized with $\mathrm{PIP}_{2}$ (Whorton and MacKinnon, 2011). This mutation would also be expected to disrupt the Arg-201/Asp-228 salt-bridge interaction, unmasking the negatively charged Asp-228 thought to be inhibitory to channel activity, presumably by engaging the critical R230 residue (Zhang et al., 1999; RosenhouseDantsker et al., 2008). Consistent with this idea, the Kir3.2*_D228N potentiated Kir3.2* currents approximately two-fold [ $n=7$ for each] (Fig. 8A). Moreover, we found that, in the Kir3.2* background, the R201A mutant produced very small currents compared with the control channel, which were nearly indistinguishable from background (Fig. 8B). Addition of the neutralization mutation D228N (Kir3.2*_D228N_R201A, $[n=7])$ potentiated the current $>20$-fold, but the currents were still significantly reduced compared with either D228N alone or Kir3.2* (Fig. 8B). These results suggest that mutation of R201 is detrimental to channel activity and that the negatively charged Asp-228 also greatly reduces channel activity. Futhermore, in Kir3.2*_R201A recordings from insideout macropatches, we were unable to stimulate the current with sodium, as has been shown with wild-type Kir3.2 and in Kir3.2* in our hands (data not shown). It is unclear whether this was because the Kir3.2*_R201A channel was not sensitive to sodium, or that there were not enough functional channels in the patch to see activity. This result further underscores the adverse effect of the R201A mutation on channel activity.

We attempted to determine whether the salt-bridge interaction between Glu-196 and Arg-201 of the S196E mutant channel could be reversed, adding further evidence to the importance of this interaction inferred from the simulation and mutation data. We constructed the mutant channels Kir3.2* R201E and Kir3.2*_S196R, which did not have detectable current. However, the double mutant Kir3.2*_S196R_R201E also did not yield detectable current. These negative results neither bolster nor refute
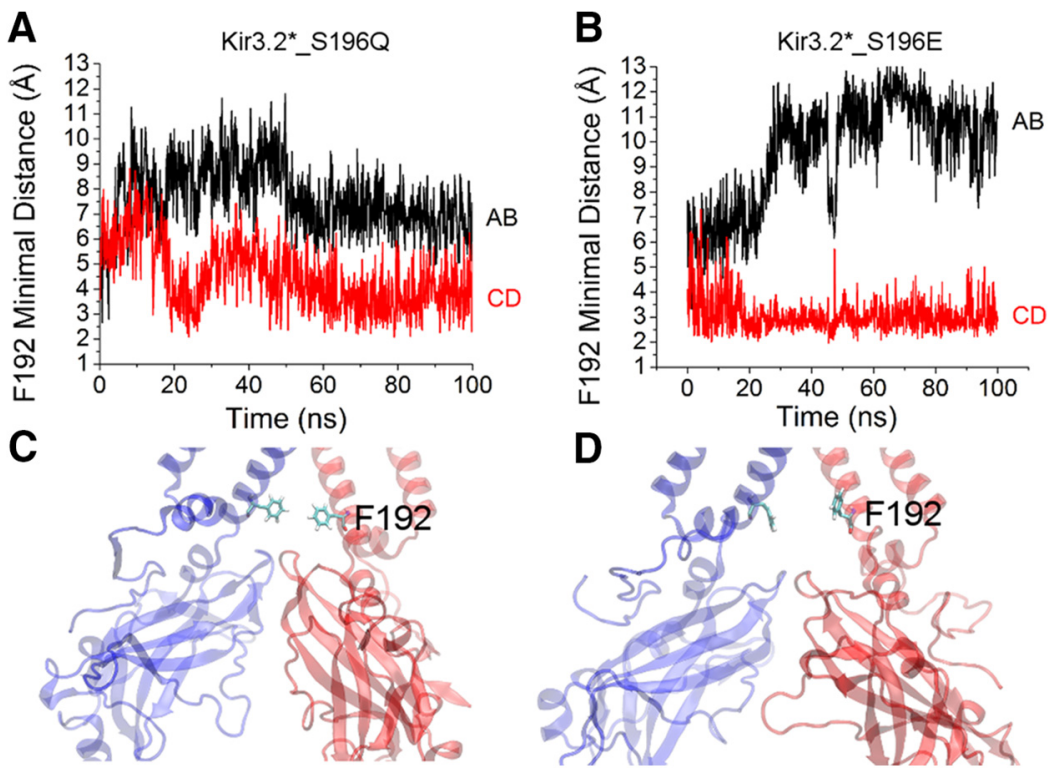

Figure 5. Intersubunit helix bundle crossing distance at F192 increases in S196E, but not S196Q. A, Time course for S196Q for $A-B$ and $C-D$ intersubunit minimal distances. $B$, Time course for $S 196$ E showing an increase in the $A-B$ distance. $C, A n 80$ ns snapshot showing the F192 A-B distance in S196Q. Chain A is colored in red and chain B is colored in blue. D, An 80 ns snapshot showing the F192 A-B distance in S196E.
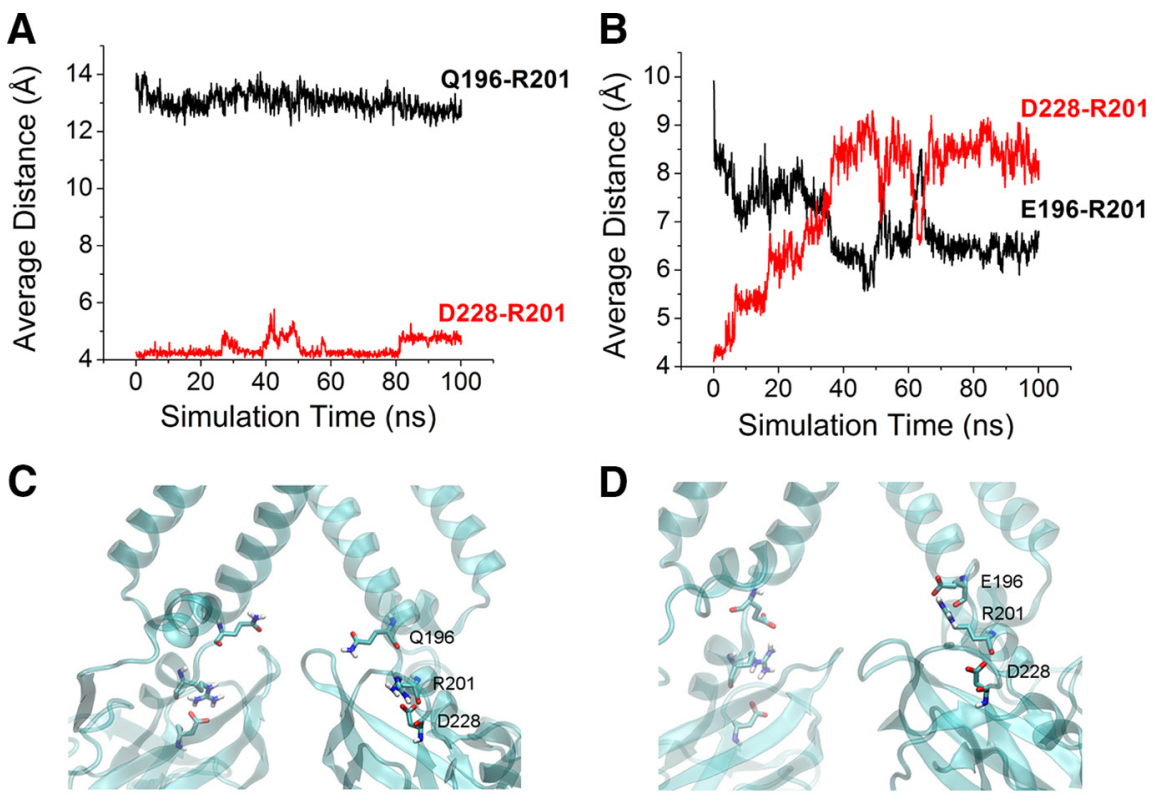

Figure 6. Comparison of simulation results for $\mathrm{S} 196 \mathrm{Q}$ and $\mathrm{S196 \textrm {E }}$ in Kir3.2* background. $A$, Simulation time course for average distance per subunit for Arg-201(CZ)/Gln-196(CD) and Arg-201(CZ)/Asp-228(CG). B, Time course for Arg-201(CZ)/Glu-196(CD) and Arg-201(CZ)/Asp-228(CG).C, An 80 ns snapshot of MD simulation depicting Gln-196, Arg-201, and Asp-228. D, An 80 ns snapshot depicting Glu-196, Arg-201, and Asp-228.

the hypothesized salt-bridge interaction between Arg-201 and Glu-196 (and by extension Arg-201/PhosphoSer-196) in the S196E mutant.

The Kir3.2*_S196E mutant had a profile similar to Kir3.2* of increased current level and decreased PMA inhibition, whereas the Kir3.2*_R201A and Kir3.2*_D228N_R201A mutants had reduced currents, similar to Kir3.2*_S196Q. Given that the S196Q mutant had increased PMA inhibition as well as diminished whole-cell currents, we tested the PMA inhibition of the Kir3.2*_D228N_R201A mutant, finding that it was significantly increased compared with Kir3.2*_D228N alone (47.5 $\pm 3.6 \%$ for 
A

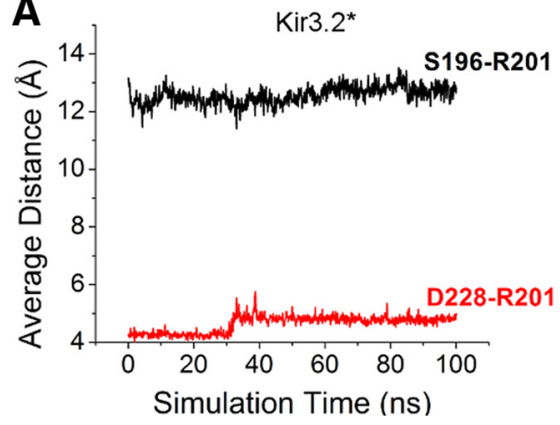

C

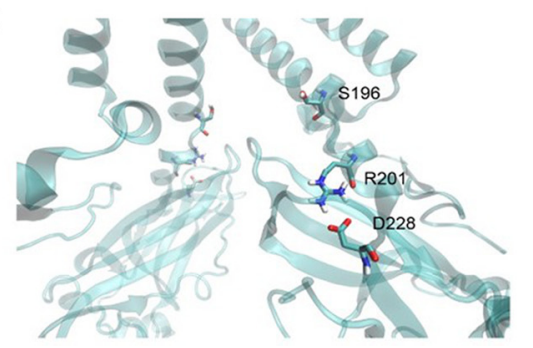

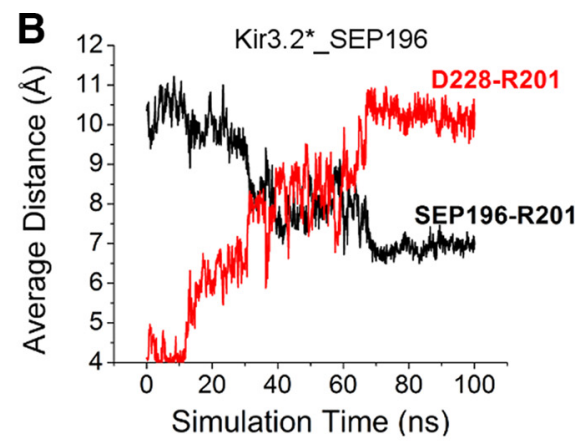

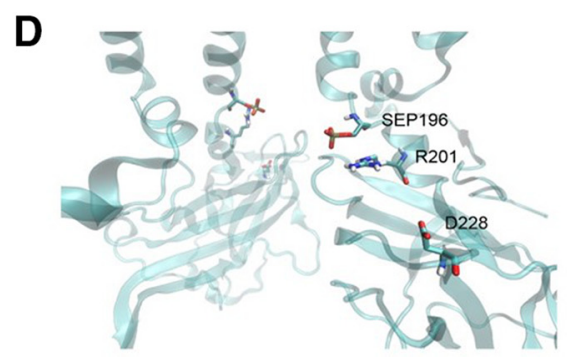

Figure 7. Comparison of simulation results for Kir3.2* and Kir3.2* _SEP196.A, Simulation time course for average distance per subunit for Ser-196(CB)/Arg-201(CZ) and Arg-201(CZ)/Asp-228(CG). B, Time course for SEP-196(P)/Arg-201(CZ) and Arg-201(CZ)/ Asp-228(CG). C, 80 ns snapshot depicting Arg-201, Ser-196, and Asp-228. D, An 80 ns snapshot depicting Arg-201, SEP196, and Asp-228.

Kir3.2*_D228N_R201A $[n=4]$ vs $19 \pm 3.9 \%$ for Kir3.2*_D228N $[n=4] ; p=0.0027$; Fig. $8 C)$. These results indicate that the putative interaction of Arg-201 with phosphorylated Ser-196 is important for both controlling channel activity and limiting PKC-mediated inhibition.

\section{Discussion}

The present study combined experimental evidence with simulations to investigate how channel activity is controlled at a putative PKC phosphorylation site, Ser-196, first identified in homologous Kir3 channel subunits. Surprisingly, we demonstrated that Ser-196 exerts allosteric control over PKC inhibition of Kir3.2, rather than being itself a direct target of PKC phosphorylation. We identified a triad residue interaction in the Kir3.2 channel, where the key players are Ser-196, Arg-201, and Asp-228. Phosphorylation of Ser-196 presents a toggle switch that recruits Arg-201 away from Asp-228 (Fig. 9). This in turn results in increased channel activation and lower sensitivity to PMA inhibition. Disruption of the SEP196/Arg-201 interaction, either by S196Q or R201A, results in a channel with greatly reduced currents, as well as higher sensitivity to PMA inhibition. Interestingly, the destabilization of the interaction between Arg-201 and phosphoSer-196 appears to affect $\mathrm{PKC}$ inhibition independently of $\mathrm{PIP}_{2}-$ channel interactions. In $\mathrm{PIP}_{2}$ reduced states (i.e., wortmannin pretreatment), the Kir3.2* channel had increased inhibition regardless of the mutation at Ser-196. Even increasing channel-PIP ${ }_{2}$ interactions, as in the $\mathrm{I} 234 \mathrm{~L}$ mutant, failed to affect the residue dependence at Ser-196 on PKC inhibition. Multiple studies to date have demonstrated that the extent of PKC inhibition depends on $\mathrm{PIP}_{2}$, including in the native rat hippocampal channels activated by baclofen (Sohn et al., 2007a, 2007b). These data indicate that the residue triad identified in Kir3.2 operates independently of $\mathrm{PIP}_{2}$, at least in terms of PKC inhibition.

When Ser-196 is phosphorylated, the helix bundle-crossing gate is stabilized and the channel is rendered less sensitive to PKC inhibition. Phosphorylated Ser-196 competes with Asp-228 for the critical Arg-201. In the absence of phosphorylation, Asp-228 interacts strongly with Arg-201, stabilizing the closed state. Interestingly, the R201A mutant has been used to obtain the crystal structure of Kir3.2 with an open G-loop gate. In the presence of $\mathrm{PIP}_{2}$, this mutant is thought to have a partially open helix bundle-crossing gate as well. Presumably due to packing of crystal contacts, only two $\mathrm{PIP}_{2}$ molecules are bound per tetramer, such that one monomer has an open helix bundle crossing and its partner maintains a $\mathrm{PIP}_{2}$-deficient structure. Therefore, the R201A mutant has been described as a constitutive, fully active channel (Whorton and MacKinnon, 2011). However, the current levels of Kir3.2*_R201A are drastically reduced compared with the Kir3.2* control. Neutralization of Asp-228 with the D228N mutant rescues Kir3.2*_R201A currents more than 20-fold, much greater than the effect of D228N alone on Kir3.2* (approximately 2-fold). The Kir3.2* D228N_R201A double mutant has increased sensitivity to PMA inhibition compared with the Kir3.2*_D228N control, again suggesting that the R201A mutant reduces allosterically channel-PIP 2 interactions (Zhang et al., 1999).

To demonstrate the existence of a salt-bridge interaction, sometimes the two interacting residues can be switched, as previously demonstrated in Kir3.4* interacting with G $\beta \gamma$ (Mahajan et al., 2013). The mutants constructed for the salt-bridge residue exchange experiment (Kir3.2*_S196R, Kir3.2*_R201E, and Kir3.2*_S196R_R201E) did not yield measurable current, which neither lent support nor refuted the salt-bridge hypothesis between phosphoSer-196 and Arg-201. The mutant Kir3.2_R201E was previously constructed in Whorton and MacKinnon (2011) and did not conduct current in the wild-type channel background either.

The potentiation effect of the D228N mutation on Kir3.2*_R201A indicates that Asp-228 likely inhibits the channel in the absence of the Arg-201 side-chain. Asp-228 is a critical residue for sodium potentiation, whereby sodium binding shields the negatively charged aspartate from positive $\mathrm{PIP}_{2}$-interacting residues (Ho and Murrell-Lagnado, 1999; Zhang et al., 1999; Rosenhouse-Dantsker et al., 2008; Inanobe et al., 2010; Whorton and MacKinnon, 2011). We were unable to demonstrate stimulation of the Kir3.2*_R201A mutant channel with sodium, likely because the channel had very low activity to begin with; the Kir3.2* channel displayed the characteristic sodium sensitivity similar to the wild-type channel, which is abrogated by the D228N mutation (as in Inanobe et al., 2010). A likely candidate for interaction with Asp-228 is Arg-230, located two residues away on the $\mathrm{CD}$ loop. Simulations in a homology model of Kir3.4 indicate that sodium coordination prevents interaction between Asp-223 and Arg-225 in Kir3.4, the homologous residues to Asp-228 and Arg-230 (RosenhouseDantsker et al., 2008). In the cocrystal of Kir3.2 and PIP Arg-230 does not form interactions with $\mathrm{PIP}_{2}$, but it is possible that Arg-230 interacts with Asp-228 in some intermediate $\mathrm{PIP}_{2}$-binding state. Evidence from $\mathrm{MD}$ simulations in the 

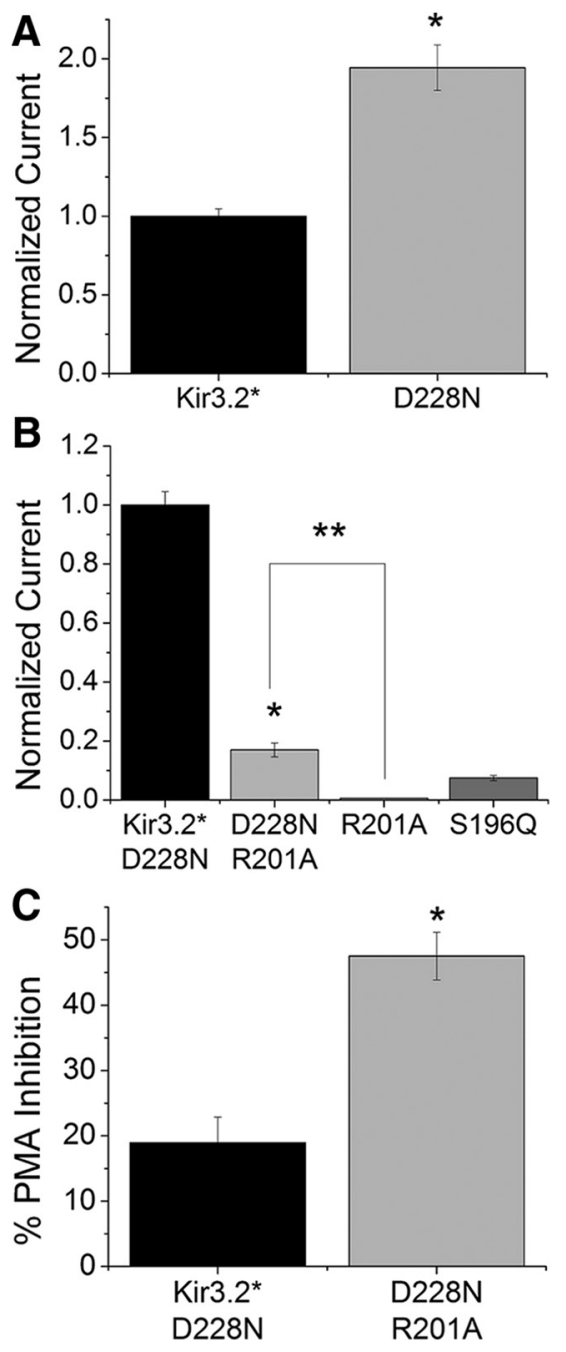

Figure 8. Neutralization of R201 results in low currents with increased sensitivity to PMA in the Kir3.2*_D228N background. A, Kir3.2* DD228N has higher currents than Kir3.2* control. B, Kir3.2*_R201A_D228N has reduced currents compared with Kir3.2* D228N. **, Kir3.2* R201A_D228N has significantly higher currents than Kir3.2* R201A at $p<0.05$. C, PMA inhibition is increased in Kir3.2*_D228N_R201A compared with Kir3.2*_D228N control. ${ }^{*} p<0.05$ compared with control.

Kir3.1-chimera structure points to this possibility, where the equivalent residue (Arg-230 equivalent) makes $\mathrm{PIP}_{2}$ contacts in the dilated (G-loop open) conformation only (Meng et al., 2012).

The mutagenesis data regarding current levels and PMA inhibition suggest that Ser-196 is normally phosphorylated in Kir3.2 expressed in Xenopus oocytes. In support of this idea of constitutive phosphorylation, we found that purified Kir3.2 protein was subject to phosphorylation, as evidenced by the alkaline phosphatase treatment assay. This assay looked at the global phosphorylation of the protein and was not specific for Ser-196; however, other residues are likely involved in the constitutive pattern of phosphorylation. In the absence of phosphorylation at Ser-196, it seems unlikely that Ser-196 (an uncharged, polar residue) would function equivalently to Glu-196 (a negatively charged residue). Because the principal difference between Gln and Glu is the introduction of negative charge, we discern that Ser-196 is likely in a phosphorylated state.

It remains to be determined whether Kir3.1 and/or Kir3.4 also might be constitutively phosphorylated at this posi-

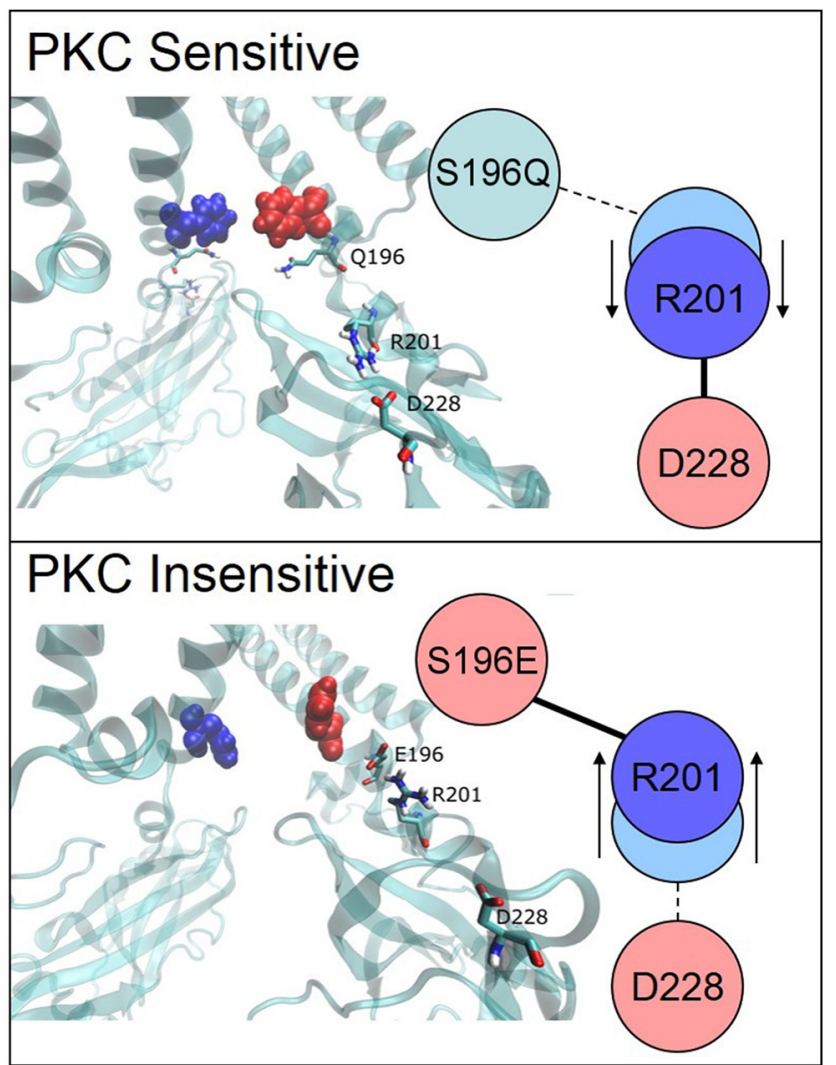

Figure 9. Schematic of PKC inhibition depending on phosphorylation state of Ser-196 and salt-bridge formation with Arg-201. Top, In PKC-sensitive channels, Q196 or dephosphorylated S196 cannot interact with R201 and are stabilized by D228. Bottom, In PKC-insensitive channels, R201 interacts with E196 or phosphorylated S196, stabilizing the open state of the helix-bundle-crossing gate.

tion, confounding the mutagenesis results on $\mathrm{PKC}$ inhibition in Mao et al. (2004). A contrasting pattern of PKC inhibition is seen with the mutagenesis of Kir3.4* compared with Kir3.2*: the neutralization mutant Kir3.4*_S191A decreases PKC inhibition, whereas the similar mutant in Kir3.2*_S196Q increases PKC inhibition. We do not claim that the critical serine residue in Kir3.4* behaves in the same manner as in Kir3.2*, but given the similarity of the two channels, an allosteric role for Ser-191 in PKC inhibition cannot be ruled out. We hope that this study will stimulate structural correlates of PKC inhibition in Kir3.1 and Kir3.4 as these structures become available.

In summary, we have highlighted that the residue Ser-196 in Kir3.2 is involved in conferring PKC-mediated inhibition to the channel. This residue was previously identified in Kir3.1 and Kir3.4 as a target of PKC phosphorylation, but biochemical confirmation has so far been lacking. Contrary to its proposed action in Kir3.1/3.4, our data demonstrate that the charge of this residue mediates allosteric control of whether Kir3.2 channels are subject to PKC-mediated inhibition. When this residue is neutralized, the inhibition of Kir3.2 is increased, likely by disrupting the interaction of phosphoSer-196 with Arg-201. Similarly, when Arg201 is neutralized, PKC-mediated inhibition is also increased. The decreased currents of either the S196Q or the R201A mutants suggest that these channels are less functional. In light of these data, the suggestion that $\mathrm{R} 201 \mathrm{~A}$ represents a constitutively active channel seems unlikely. 


\section{References}

Brown SG, Thomas A, Dekker LV, Tinker A, Leaney JL (2005) PKC-delta sensitizes Kir3.1/3.2 channels to changes in membrane phospholipid levels after M3 receptor activation in HEK-293 cells. Am J Physiol Cell Physiol 289:C543-C556. CrossRef Medline

Hansen SB, Tao X, MacKinnon R (2011) Structural basis of PIP2 activation of the classical inward rectifier K+ channel Kir2.2. Nature 477:495-498. CrossRef Medline

Hibino H, Inanobe A, Furutani K, Murakami S, Findlay I, Kurachi Y (2010) Inwardly rectifying potassium channels: their structure, function, and physiological roles. Physiol Rev 90:291-366. CrossRef Medline

Ho IH, Murrell-Lagnado RD (1999) Molecular mechanism for sodiumdependent activation of G protein-gated K+ channels. J Physiol 520:645651. CrossRef Medline

Huang CL, Feng S, Hilgemann DW (1998) Direct activation of inward rectifier potassium channels by PIP2 and its stabilization by gbetagamma. Nature 391:803-806. CrossRef Medline

Inanobe A, Yoshimoto Y, Horio Y, Morishige KI, Hibino H, Matsumoto S, Tokunaga Y, Maeda T, Hata Y, Takai Y, Kurachi Y (1999) Characterization of G-protein-gated $\mathrm{K}+$ channels composed of Kir3.2 subunits in dopaminergic neurons of the substantia nigra. J Neurosci 19:1006-1017. Medline

Inanobe A, Nakagawa A, Matsuura T, Kurachi Y (2010) A structural determinant for the control of PIP2 sensitivity in G protein-gated inward rectifier K+ channels. J Biol Chem 285:38517-38523. CrossRef Medline

Keselman I, Fribourg M, Felsenfeld DP, Logothetis DE (2007) Mechanism of PLC-mediated Kir3 current inhibition. Channels (Austin) 1:113-123. CrossRef Medline

Kobrinsky E, Mirshahi T, Zhang H, Jin T, Logothetis DE (2000) Receptormediated hydrolysis of plasma membrane messenger PIP2 leads to $\mathrm{K}+$ current desensitization. Nat Cell Biol 2:507-514. CrossRef Medline

Leaney JL, Dekker LV, Tinker A (2001) Regulation of a G protein-gated inwardly rectifying $\mathrm{K}+$ channel by a $\mathrm{Ca}(2+)$-independent protein kinase C. J Physiol 534:367-379. CrossRef Medline

Logothetis DE, Kurachi Y, Galper J, Neer EJ, Clapham DE (1987) The beta gamma subunits of GTP-binding proteins activate the muscarinic K+ channel in heart. Nature 325:321-326. CrossRef Medline

Lopes CM, Zhang H, Rohacs T, Jin T, Yang J, Logothetis DE (2002) Alterations in conserved kir channel-PIP2 interactions underlie channelopathies. Neuron 34:933-944. CrossRef Medline

Mahajan R, Ha J, Zhang M, Kawano T, Kozasa T, Logothetis DE (2013) A computational model predicts that $\mathrm{G} \beta \gamma$ acts at a cleft between channel subunits to activate GIRK1 channels. Sci Signal 6:ra69. CrossRef Medline

Mao J, Wang X, Chen F, Wang R, Rojas A, Shi Y, Piao H, Jiang C (2004) Molecular basis for the inhibition of $\mathrm{G}$ protein-coupled inward rectifier $\mathrm{K}(+)$ channels by protein kinase C. Proc Natl Acad Sci U S A 101:10871092. CrossRef Medline

Meng XY, Zhang HX, Logothetis DE, Cui M (2012) The molecular mecha- nism by which PIP(2) opens the intracellular G-loop gate of a Kir3.1 channel. Biophys J 102:2049-2059. CrossRef Medline

Pattnaik BR, Asuma MP, Spott R, Pillers DA (2012) Genetic defects in the hotspot of inwardly rectifying $\mathrm{K}(+)$ (kir) channels and their metabolic consequences: A review. Mol Genet Metab 105:64-72. CrossRef Medline

Rosenhouse-Dantsker A, Sui JL, Zhao Q, Rusinova R, Rodríguez-Menchaca AA, Zhang Z, Logothetis DE (2008) A sodium-mediated structural switch that controls the sensitivity of kir channels to $\operatorname{PtdIns}(4,5) \mathrm{P}(2)$. Nat Chem Biol 4:624-631. CrossRef Medline

Sohn JW, Lee D, Cho H, Lim W, Shin HS, Lee SH, Ho WK (2007a) Receptor-specific inhibition of GABAB-activated $\mathrm{K}+$ currents by muscarinic and metabotropic glutamate receptors in immature rat hippocampus. J Physiol 580(Pt. 2):411-422.

Sohn JW, Lim A, Lee SH, Ho WK (2007b) Decrease in PIP(2) channel interactions is the final common mechanism involved in PKC- and arachidonic acid-mediated inhibitions of GABA(B)-activated $\mathrm{K}+$ current. J Physiol 582(Pt 3):1037-1046.

Stevens EB, Shah BS, Pinnock RD, Lee K (1999) Bombesin receptors inhibit G protein-coupled inwardly rectifying $\mathrm{K}+$ channels expressed in xenopus oocytes through a protein kinase C-dependent pathway. Mol Pharmacol 55:1020-1027. Medline

Torrecilla M, Marker CL, Cintora SC, Stoffel M, Williams JT, Wickman K (2002) G-protein-gated potassium channels containing Kir3.2 and Kir3.3 subunits mediate the acute inhibitory effects of opioids on locus ceruleus neurons. J Neurosci 22:4328-4334. Medline

Vivaudou M, Chan KW, Sui JL, Jan LY, Reuveny E, Logothetis DE (1997) Probing the G-protein regulation of GIRK1 and GIRK4, the two subunits of the KACh channel, using functional homomeric mutants. J Biol Chem 272:31553-31560. CrossRef Medline

Whorton MR, MacKinnon R (2011) Crystal structure of the mammalian GIRK2 $\mathrm{K}+$ channel and gating regulation by G proteins, PIP2, and sodium. Cell 147:199-208. CrossRef Medline

Whorton MR, MacKinnon R (2013) X-ray structure of the mammalian GIRK2-betagamma G-protein complex. Nature 498:190-197. CrossRef Medline

Yi BA, Lin YF, Jan YN, Jan LY (2001) Yeast screen for constitutively active mutant G protein-activated potassium channels. Neuron 29:657-667. CrossRef Medline

Zhang H, He C, Yan X, Mirshahi T, Logothetis DE (1999) Activation of inwardly rectifying $\mathrm{K}+$ channels by distinct PtdIns(4,5)P2 interactions. Nat Cell Biol 1:183-188. CrossRef Medline

Zhang H, Craciun LC, Mirshahi T, Rohács T, Lopes CM, Jin T, Logothetis DE (2003) PIP(2) activates KCNQ channels, and its hydrolysis underlies receptor-mediated inhibition of M currents. Neuron 37:963-975. CrossRef Medline

Zhang L, Lee JK, John SA, Uozumi N, Kodama I (2004) Mechanosensitivity of GIRK channels is mediated by protein kinase C-dependent channelphosphatidylinositol 4,5-bisphosphate interaction. J Biol Chem 279: 7037-7047. Medline 